Revue européenne des sciences sociales

European Journal of Social Sciences

XXXVIII-118| 2000

Limites de l'éthique dans l'action politique

\title{
Environnement, morale et politique
}

\section{Dominique Bourg}

\section{OpenEdition}

\section{Journals}

Édition électronique

URL : http://journals.openedition.org/ress/682

DOI : $10.4000 /$ ress.682

ISSN : 1663-4446

\section{Éditeur}

Librairie Droz

\section{Édition imprimée}

Date de publication : 1 août 2000

Pagination : 7-14

ISBN : 2-600-00450-5

ISSN : 0048-8046

Référence électronique

Dominique Bourg, «Environnement, morale et politique », Revue européenne des sciences sociales [En ligne], XXXVIII-118 | 2000, mis en ligne le 15 décembre 2009, consulté le 30 avril 2019. URL : http:// journals.openedition.org/ress/682; DOI : 10.4000/ress.682 


\section{ÉTHIQUE ET POLITIQUES DE LA TECHNO-NATURE À PROPOS DE LA BIOLOGIE DE LA CONSERVATION*}

\section{1. - UNE NOUVELLE PRATIQUE DE L'ÉCOLOGIE SAVANTE}

Les remarques qui suivent doivent être lues comme une première tentative pour construire un cadre d'interprétation sociologique destiné à rendre compte de l'émergence d'un ensemble de pratiques scientifiques regroupées sous le label de l'écologie de la restauration. L'écologie entretient des rapports complexes avec les sciences sociales: justement définie comme «la plus humaine des sciences de la nature $»^{1}$, elle ne cesse de convoquer, sous diverses formes et parfois au prix de multiples réélaborations, des éléments de savoir historique, anthropologique, sociologique ou économique. Parallèlement, les écologistes scientifiques articulent leurs programmes de recherche à une triple problématique de l'urgence sociale, de l'aménagement rationnel du territoire et du développement durable: l'immersion de leur savoir disciplinaire dans la vie de la cité est un trait constitutif de leur activité. Le domaine appliqué que constitue l'écologie de la restauration offre un terrain privilégié pour étudier les formes d'inscription territoriale et sociale qui caractérisent le développement de ce type de savoir.

Les entreprises de restauration, de réhabilitation, voire de recréation d'écosystèmes ont connu un développement notable aux cours de ces dernières années: bien que la définition et l'ampleur des projets varient considérablement, et que le développement de ces pratiques soit très contrasté en fonction des conjonctures nationales (qu'il s'agisse de leur dimension scientifique ou institutionnelle), la dynamique en cours est particulièrement frappante, plus encore peut-être par les débats qu'elle suscite que par les opérations de terrain qu'elle engage. L'écologie de la restauration apparaît comme une sous-discipline en croissance rapide dans le champ de la biologie de la conservation: il s'agit de réhabiliter ou de rétablir des espèces, des populations et des systèmes dégradés ou condamnés par les usages sociaux multiples des ressources naturelles accumulées à travers 1'histoire. Les objets que traite cette sous-discipline se situent à l'intersection de préoccupations économiques et sociales et de préoccupations biologiques. Préoccupations sociales d'abord: les objectifs écosystémiques ne peuvent être dissociés de l'em-

* Ce texte s'appuie sur une étude financée par le service de la recherche et des affaires économiques du ministère de l'Environnement. Je remercie Geneviève Barnaud, Véronique Barre et Martine Berlan pour leur soutien.

1 J.-P. Deléage, Histoire de l'écologie. Une science de l'homme et de la nature, Paris, La Découverte, 1991 . 
prise spatiale différentielle des activités économiques, de la constitution progressive d'une politique de gestion des ressources naturelles, des formes contrastées que prend la demande sociale d'espaces récréatifs et des transformations qui affectent les définitions sociales de la nature. Préoccupations biologiques ensuite: celles-ci présentent à leur tour un caractère croisé: nous sommes à l'évidence dans le cadre d'une mission-oriented science, qui implique le développement d'une ingénierie appropriée et la constitution de savoir-faire appliqués ${ }^{2}$. Simultanément, le type d'intervention sur le terrain qu'exige l'écologie de la restauration fournit une opportunité pour éprouver la validité des théories écologiques fondamentales $^{3}$. Le territoire d'une éco-technologie appliquée constitue un espace de test inédit pour les théories, espace dont certains pensent qu'il doit être privilégié du fait des expérimentations «en vraie grandeur» qu'il permet.

L'impératif de conservation n'est jamais la traduction pure et simple de normes biologiques: il s'agit inévitablement du produit d'une négociation sur la place à accorder aux valeurs «naturelles» dans les sociétés contemporaines. Le débat porte sur les moyens de déterminer la valeur économique des biens naturels ou sur la capacité de les soustraire à toute forme de calcul économique au nom de principes supérieurs. Comme le fait remarquer Joël Brown, l'écologie de la restauration est presque toujours confrontée à des stratégies concurrentes d'occupation de l'espace: dans la pratique, c'est lorsque les usages alternatifs n'offrent pas d'attrait social ou économique particulier que la restauration est préférée ${ }^{4}$.

L'écologie de la restauration apparât donc comme un domaine largement ouvert aux controverses. Il existe un premier type de controverse, interne à la discipline: quand le biologiste doit-il intervenir, et avec quelle puissance d'intervention? Quelle doit être la durée de son action? Quels sont les critères d'une intervention réussie? Quels sont les indicateurs biologiques qui permettent de déterminer avec précision l'objectif à atteindre? Quels sont, si l'on considère le caractère récent du développement de ces techniques, les critères d'évaluation qui permettent d'affirmer que l'objectif a été durablement atteint? On connaît surtout les controverses qui ont lieu à propos de la réintroduction d'espèces animales, mais il en existe bien d'autres, moins visibles mais qui portent le plus souvent sur la détermination du caractère indigène («native») ou historique d'un élément du système ou de l'ensemble du système. Ces controverses savantes peuvent être reprises et relayées dans des cercles plus larges: les prises de position contradictoires des biologistes peuvent être mobilisées comme des ressources pour un deuxième type de controverse, qui se développe dans le monde social, à propos de la légitimité de ces interventions restauratrices. A ce titre la branche de la biologie de la conservation sur laquelle nous portons notre attention constitue une excellente illustration du haut degré d'incertitude qui caractérise le contexte de la décision en matière de protection. Aux questions épistémologiques que suscite le sta-

2 M. L. Bowles \& C. J. Whelan. Restoration of Endangered Species. Conceptual Issues, Planning and Implementation, Cambridge, Cambridge University Press, 1996.

3 W. R. III Jordan, M. E. Gilpin \& J. D Aber, Restoration Ecology. A Synthetic Approach to Ecological Research, Cambridge, Cambridge University Press, 1987.

4 J. S. Brown, Restoration ecology: Living with the Prime Directive, in M. L. Bowles and C. J. Whelan, op. cit., p. 357. 
tut particulier de la discipline (comme en témoigne le caractère jugé quelquefois «peu robuste » de nombre de ses assertions) s'ajoutent le débat éthique concernant la légitimité d'une intervention réparatrice et la référence à l'esthétisation de la nature qui conduit à une hiérarchie des formes de spectacle qu'elle peut offrir.

Dans le cas de l'écologie de la restauration définie comme contribution de la science aux techniques de gestion d'un espace visant à « redonner à un écosystème endommagé les conditions antérieures à la perturbation $»^{5}$ aussi bien que dans celui de la biologie de la conservation qui l'englobe, lorsqu'on la définit comme «instrument d'évaluation de l'impact des actions de l'homme sur les systèmes naturels et de développement de solutions appropriées pour éviter l'extinction des populations et des espèces ${ }^{6}$, il s'agit bien de proposer une vision instrumentale de l'activité savante. L'espace peut désormais faire l'objet d'une forme de gestion rationnelle.

Il n'est pas indifférent que la problématique de la contribution évaluatrice ou instrumentale de la biologie soit souvent associée au progrès de la science. Ainsi Jacques Blondel place l'émergence de la biologie de la conservation sous le double signe de la crise écologique (qui s'exprime avec le constat de plus en plus argumenté de la diminution «alarmante» de la diversité biologique) et du progrès «théorique» de l'écologie. J. Blondel exprime dans la même page cette avancée conceptuelle sous deux formes, qu'il vaut la peine de distinguer. La première est caractérisée par «le développement d'une véritable écologie scientifique qui la démarqua définitivement de «l'Histoire Naturelle» dont elle est l'héritière». La seconde est marquée par le fait que «plusieurs courants caractérisent le développement de la Biologie de la Conservation en tant que discipline scientifique dotée d'un arsenal méthodologique et conceptuel qui la distingue de la Protection de la Nature traditionnelle $»^{7}$. Nous avons donc affaire, dans cette dynamique scientifique, à l'émergence d'une double démarcation: l'histoire naturelle est définitivement renvoyée au passé de la discipline, à un style scientifique et à une série de pratiques sociales définies comme révolues. La protection de la nature, comme préoccupation sociale et institutionnelle, telle qu'elle s'exprime dans l'affirmation d'un goût pour la nature et de dispositions réglementaires, est également renvoyée à une tradition désormais obsolète. L'auteur est néanmoins conduit à nuancer fortement ses affirmations lorsqu'il reconnaît, à la fin de son article, «qu'une des grandes difficultés de l'application concrète de mesures de conservation est le fossé entre l'urgence des mesures à prendre et la fragilité de l'assise scientifique $\gg^{8}$.

Le point de vue exprimé par ce chercheur a le grand intérêt de mettre au jour la situation paradoxale de toutes les entreprises savantes qui utilisent la bannière de la conservation et de la restauration: il s'agit simultanément de disqualifier les pratiques «traditionnelles » de gestion ou de protection au motif qu'elles n'ont pas

5 G. Barnaud, A l'interface de la pratique et de la théorie : l'écologie de la restauration, «Natures, sciences, sociétés », 1994, vol. 3, pp. 36-50.

6 J. Blondel, Du théorique au concret : la biologie de la conservation, «Natures, sciences, sociétés », 1995, vol. 3, pp. 10-18.

7 J. Blondel, op.cit., p. 10.

$8 \quad$ J. Blondel, op.cit., p.16. 
d'assise scientifique et d'exemplifier continuellement le constat de l'écart entre le stock de connaissances scientifiques disponibles et la complexité des situations concrètes. Ce double mouvement, offre de services, voire prosélytisme savant d'un côté, affirmation d'une nécessaire modestie scientifique de l'autre, a pour effet d'accroître, au moins dans le monde social, le caractère incertain des énoncés de l'écologie, au moment même où celle-ci, par les ruptures ostentatoires qu'elle multiplie avec son passé disciplinaire et avec les définitions profanes de la gestion de la nature, fait valoir de nouvelles compétences. En d'autres termes, les biologistes qui travaillent dans le secteur de l'écologie proclament à la fois leurs avancées théoriques (ce que Blondel appelle suggestivement «arsenal conceptuel») en tant qu'elles sont liées à la disqualification de pratiques (qu'il s'agisse de pratiques savantes, comme les opérations de collection et de classification de l'histoire naturelle, elles-mêmes fortement liées à des pratiques d'amateur, ou de pratiques sociales liées à la gestion pratique ou bureaucratique des espaces et des espèces) et l'intrication entre le savoir théorique nouvellement produit et des nouvelles pratiques de gestion qui excèdent pourtant les cadres du travail scientifique. Bien des formes de présentation de soi ou de justification de l'activité de recherche sont l'effet de la tension entre l'ambition théorique et les contraintes inédites qu'impose l'affiliation à des formes variées de pratique gestionnaire.

Les questions sont présentes, plus ou moins explicitement, dans toutes les réflexions des écologues eux-mêmes sur ce nouvel aspect de leur pratique. Le développement de l'écologie de la restauration a lieu dans un contexte de transformation de l'attitude des écologistes savants à l'égard de ce qu'on pourrait appeler « intervention sur le social». On constate d'abord la prise en compte croissante par les écologues, à travers notamment la notion d'écosystème modifié, des usages sociaux des ressources naturelles. Bernadette Lizet a montré, à propos des «brouteurs archaïques» comment on était passé d'une éthique du non-interventionnisme à une perspective gestionnaire. «L'idée et la stratégie de la «réserve intégrale » (sans aucune intervention humaine) ont été radicalement contestées par un nouveau courant de recherches écologiques expérimentales, conduites sur le territoire (sur les «sites», pour reprendre l'expression consacrée)... Si l'activité pastorale «douce» - c'est-à-dire «traditionnelle»-, vient à faire défaut, le relais doit être pris (et il l'est) par de nouvelles pratiques d'entretien écologique de l'espace, voire de «restauration». Le respect de la nature, principe quasi-sacralisé de non-interventionnisme, s'est donc inversé en parti-pris gestionnaire ${ }^{9}$. L'impératif de gestion trouve aussi de nouvelles justifications dans l'émergence d'une problématique de la bio-diversité. Valentin Pelosse et André Micoud font justement remarquer que «la diversité biologique, alias bio-diversité, est devenue un slogan de la revendication de gestion écologique $»^{10}$.

Bernadette Lizet ajoute que ce déplacement est l'occasion de la constitution d'une nouvelle culture professionnelle, qui associe les savoirs de l'écologie aux impératifs de la gestion. Parallèlement à cette transformation du rapport au métier

19 B. Lizet, Les brouteurs archä̈ques du génie écologique, in «Crise de l'environnement, éthique, science et politique», colloque CREA-STEPE, multigraphié, 1994.

10 V. Pelosse et A. Micoud, Du domestique au sauvage: des catégories pertinentes de la biodiversité, «Etudes rurales», 1993, n 129-130, pp. 9-14. 
qu'induit l'extension du génie écologique, il faut noter que la notion de patrimoine à protéger s'élargit aux objets naturels qui apparaissent comme les produits historiques de pratiques sociales (le pastoralisme et la sylviculture en constituent les meilleurs exemples): la nature primitive ou inviolée ne constitue plus la norme unique de la justification du geste protecteur ou patrimonial. Bien plus, par une sorte d'effet en retour, les morceaux de nature dans lesquels ne se sont jamais exercées d'activités sociales ayant des effets sur les équilibres naturels font aussi l'objet d'une intervention gestionnaire, comme si le modèle de l'utilisateur respectueux (qui sous-tend les représentations des activités douces ou traditionnelles dont parle Bernadette Lizet) pouvait être exporté en direction de la nature sans utilisateur, susceptible à son tour de faire l'objet d'une gestion rationnelle.

Une telle mutation de la définition légitime de la posture savante par rapport à la nature (reconnaissance des mérites écologiques de la nature «doucement» transformée par l'activité humaine, apparition d'un impératif de gestion écologique étendu jusqu'aux confins de la «nature pure») constitue, sans qu'on l'ait toujours clairement remarqué, l'origine des nouvelles interrogations éthiques des écologistes scientifiques. Le non-interventionnisme ne posait pas de difficultés, puisqu'il s'agissait, armé d'indiscutables savoirs concernant le fonctionnement des écosystèmes, d'entourer la vraie nature d'une sorte de périmètre de sécurité afin de la laisser être ce qu'elle est. L'opposition entre l'humain (saisi à travers la figure de la prédation de ressources ou celle, plus complexe, de la domestication) et le naturel (identifié simplement au sauvage ou à l'auto-produit) avait la fonction d'un grand partage qui assignait à l'écologue le soin de défendre la nature contre ses utilisateurs profanes: l'impératif éthique d'engagement dans le domaine de la protection de la nature était le simple effet de la traduction d'une compétence savante.

Prenons comme exemple cette phrase tirée de l'ouvrage récent d'un chercheur doublement engagé, dans la science et dans la lutte écologique: "L'humanisation de la nature ne donne pas deux fois sa chance au monde sauvage », écrit Jean-Paul Deléage $^{11}$. Dans ce cas, les choses sont simples: il y a un avant et un après de l'humanisation. Or, la restauration de la nature revient à donner au sauvage une deuxième chance, la possibilité d'une réversibilité. Il ne s'agit plus d'arrêter ou d'empêcher un processus d'anthropisation, mais de remonter le cours du temps de l'anthropisation à l'aide de dispositifs anthropiques. L'écologie de la restauration vient donc brouiller la ligne de partage établie entre le naturel et le social et entre le sauvage et le domestique. Le développement du génie écologique pose de surcroît le problème de la spécificité d'une intervention scientifiquement armée par rapport à d'autres interventions sur la nature (celle du paysagiste, qui peut être étroitement associé à l'écologue dans la définition et la mise en œuvre d'une opération de terrain, mais aussi celle de tous les acteurs sociaux qui mobilisent des savoirs pratiques sur les mêmes terrains ${ }^{12}$ ). La restauration constitue en effet une épreuve inédite pour l'écologue, qui doit justifier la spécificité de sa compétence dans des termes nouveaux. Alors qu'une posture de protecteur de la nature pure

J.-P. Deléage, op.cit., p. 220.

12 J.-L. Fabiani, L'écologie de la restauration considérée comme mise en spectacle du patrimoine naturel, «Carnets du paysage $, 1999, \mathrm{n}^{\circ} 4, \mathrm{pp} .80-95$. 
met en jeu très clairement la coupure entre savant et profane, entre protecteur et prédateur, celle du restaurateur conduit à composer avec différents ordres de savoir. La démarche de patrimonialisation de la nature conduit en outre à multiplier les zones de contamination entre patrimoine naturel et patrimoine culturel, et donc à penser différemment l'action de l'homme à l'égard du milieu naturel. De ces multiples déplacements naît l'incertitude éthique qui saisit aujourd'hui l'écologie.

Ces incertitudes caractérisent l'ensemble des questions qui touchent à l'environnement. Les zones d'insécurité éthique et juridique y abondent en effet, et chaque «avancée» dans le domaine, qu'elle prenne la forme d'injonctions morales ou de dispositions légales, contribue à engendrer de nouvelles formes d'incertitude: tout se passe comme si la mise en œuvre du savoir d'expert n'était plus susceptible de clore un débat sur un domaine déterminé de l'action humaine par la mise en œuvre de normes nouvelles. François Ewald fait justement remarquer que la généralisation de l'expertise en matière d'environnement correspond à la constitution d'un espace de débat sur les valeurs et non à la production de «réponses définitives ou de certitudes finales ${ }^{13}$. C'est dans cette perspective qu'il est en droit d'affirmer que «l'expert apparait dans la disparition du souverain». L'émergence de l'environnement comme mode de problématisation de la société constitue ainsi l'expertise comme une «illusion nécessaire » et comme une «négociation infinie sur les valeurs», selon l'auteur de l'Etat-Providence. On voit bien la difficulté qui s'attache à la mise en œuvre de tels savoirs dans le monde social: alors que l'établissement de règles stabilisées devrait signifier la conclusion d'un moment de négociation ou de débat, celui-ci instaure au contraire une sorte de discussion indéfinie. La production de normes présente donc un caractère ouvertement réflexif.

\section{2. - SAVOIR ÉCOLOGIQUE OU ART DU FAUX?}

Une des premières questions d'ordre éthique est évidemment celle qui touche à la légitimité du remplacement d'éléments de nature disparus ou très fortement dégradés. En effet, tous les objets ne sont pas remplaçables sur les mêmes bases. «Si je vous prête ma montre digitale ou ma Mitsubishi et que vous perdiez ou que vous endommagiez l'une ou l'autre, vous pouvez certainement les remplacer par une montre ou une voiture de qualité identique. A un autre extrême, vous ne pouvez certainement pas remplacer ma famille si vous portez la responsabilité de sa perte, parce que je vois ma famille, et la relation que j'entretiens avec elle, comme unique», remarque le philosophe Alastair Gunn ${ }^{14}$. On peut faire cette remarque à propos de l'exemple de Gunn: si en toute rigueur les deux Mitsubishi ne sont pas identiques, leur caractère substituable tient au fait qu'elles sont fabriquées en série (et que le véhicule perdu fait actuellement l'objet d'une production). De plus, si

13 F. Ewald, L'expertise, une illusion nécessaire, in J. Theys \& B. Kalaora (dir.), La Terre outragée, Paris, Autrement, 1992, pp. 105-109.

14 A. Gunn, The Restoration of Species and Natural Environments, «Environmental Ethics», Winter, 1991, pp. 291-310. 
vous égarez la Timex que je vous ai prêtée, je ne prendrai sans doute pas ombrage si vous la remplacez par une Casio du même type parce qu'il n'y avait que ce modèle au tabac du coin. Je n'aurai pas la même réaction si vous perdez ma Rolex et que vous me la remplacez par une Swatch. Que dirait-on si le véhicule endommagé ou perdu était un véhicule de collection d'une grande rareté (une Bugatti par exemple)? Le remplacement ne serait pas possible; la restauration pourrait l'être sous certaines conditions (déterminées par l'existence d'une épave et son état: savoir s'il vaut la peine de reconstituer une voiture dépend évidemment de sa rareté et de sa valeur avant dégradation). En tout cas, il est toujours possible d'envisager un dédommagement qui, s'il ne permet pas au propriétaire de retrouver l'original, lui fournit au moins un équivalent monétaire. Qu'en est-il du caractère substituable de la valeur sentimentale d'un objet, qu'il soit unique ou de série? Cette forme d'investissement sur l'objet ne constitue pas à proprement parler une de ses propriétés: on doit donc la laisser en dehors de l'analyse.

Peut-on remplacer un élément de nature qui a été perdu à l'exemple de la Mitsubishi selon Gunn? La nature serait-elle plutôt comparable à la Bugatti que j'introduis dans la discussion? Quelles sont les limites de l'analogie entre des biens de consommation (quels que soient par ailleurs leur valeur monétaire ou leur statut social) et des biens naturels? La distinction entre la famille et la voiture est fondée sur une seule propriété: l'unicité de la première. En fait, il y a autre chose, car il existe des exemplaires uniques de voiture (certaines voitures de collection), et cela ne suffit pas à assimiler une Bugatti et l'épouse d'Alastair Gunn (même s'il existe différents dispositifs de dédommagement pour la perte d'une personne: ceux-ci ne peuvent jamais être substitués à un remplacement). Il faut introduire dans la discussion la nature des objets et non pas seulement leur caractère unique ou reproductible. Les êtres humains ne sont pas seulement des pièces uniques: ils ont aussi pour caractéristique le fait de ne pas être assimilables à un produit (même ceux dont la naissance est le produit, au moins partiel, des effets du génie génétique), puisqu'ils sont des êtres moraux. Ils sont aussi, à la différence des objets auxquels ils sont comparés pour les besoins de la démonstration, des êtres naturels. Pour Gunn, les espèces et la plupart des zones naturelles se situent «quelque part entre ces extrêmes », c'est-à-dire entre la Mitsubishi et sa femme, ce qui suppose une sorte de gradient de substituabilité des êtres et des objets. Cette définition d'une sorte d'entre-deux laisse entière la question de savoir jusqu'à quel point la nature peut être considérée comme reproductible.

Pouvons-nous mimer la reproduction des écosystèmes? Robert Elliot a posé les bases de la discussion éthique sur la restauration dans un article paru il y a quinze ans: Faking Nature reste la référence fondamentale dans ce domaine ${ }^{15}$. Elliot défend l'argument selon lequel il existe des objections décisives à la problématique de la restauration de la nature, dans la mesure où le processus perturbation/restauration, qui postule un certain type de réversibilité, ne permet pas de retrouver la valeur naturelle ou la naturalité de la nature existant antérieurement à la modification d'un espace. Un tel point de vue suppose qu'on se donne un critère pour définir la naturalité de la nature: celle-ci ne suppose pas de degrés (nous

15 R. Elliot, Faking Nature, in R. Elliot (ed.), Environmental Ethics, Oxford, Oxford University Press, 1995. 
sommes dans une logique de tout ou rien: ou bien, un espace est naturel, ou bien il a irréversiblement perdu cet attribut), puisque la seule définition opératoire de la nature est: ce qui n'a pas été modifié par l'homme. La séduction qu'exercent les espaces qu'on regroupe sous la catégorie de wilderness, la valeur esthétique qu'ils recèlent et la possibilité de ressourcement moral qu'ils offrent est indiscutablement l'effet direct de cette non anthropisation: des espaces jardinés, même lorsqu'ils présentent au moyen d'artifices des compositions dans lesquelles l'activité humaine a été complètement effacée, comme c'est le cas du parc à l'anglaise, ne sont pas en mesure de produire ces effets. «Nous accordons de la valeur à la forêt et au fleuve en partie parce qu'ils sont représentatifs du monde qui échappe à notre domination, parce que leur existence ne dépend pas de nous.» ${ }^{16}$ Les sentiments qu'inspire la nature sont d'un autre ordre que ceux que peut inspirer la vision de réalisations humaines de grande échelle (comme un barrage ou une ville).

Deux objections viennent immédiatement à l'esprit: la première, qui est aussi la plus générale, porte sur l'association entre naturalité et valeur éthique: la naturalité n'implique pas nécessairement de valeur. Elliot prend lui-même en compte cette objection en faisant remarquer que la maladie ou l'éruption volcanique, qui sont des phénomènes naturels, et qui altèrent néanmoins ce que nous valorisons dans la nature, ne contiennent pas ce type de valeur. Mais il ne va pas jusqu'au bout de son objection puisque cette concession ne le conduit pas à la remise en cause d'une définition unique de la naturalité. Or, il y a au moins ici deux formes de nature: celle qui se donne sous l'aspect d'un ordre naturel dont l'homme sait qu'il n'a pas contribué à sa constitution; celle qui apparaît comme désordre naturel ou comme pathologie. Il faut donc ajouter au caractère non-modifié de la nature envisagée par Elliot un second critère de la valorisation: celui de l'ordre. On en vient à la deuxième objection: l'affectation d'une valeur à des milieux non modifiés par l'homme doit être référée à deux types de conditionnements historiques. Les premiers portent sur la forme de nature valorisée. On sait que la valeur accordée à des paysages a beaucoup varié au cours de l'histoire et que l'appréciation de la notion de wilderness en tant que telle (c'est-à-dire en tant qu'elle exprime la manifestation d'une extériorité de la nature par rapport aux actions humaines) est à la fois récente dans l'histoire et qu'elle peut être localisée dans des conjonctures culturelles particulières (elle constitue un élément du sentiment moderne de la nature et elle apparaît dans sa formulation la plus précise comme une expression de l'histoire de la construction sociale de l'espace américain). Les seconds conditionnements portent sur la nature elle-même: sommes-nous sûrs que la valeur s'attache automatiquement à ce qui ne dépend pas de nous? La philosophie morale ne prend-elle pas trop rapidement à son compte le principe d'une extériorité de l'homme par rapport à la nature? Ne valorisons-nous pas des espaces dotés de signification (et donc susceptibles d'investissements éthiques et esthétiques) parce qu'ils sont déjà partiellement anthropisés ou anthropisables? Un tel positionnement éthique ne correspond-il pas à un état antérieur de la science écologique? La théorie de la valeur de la naturalité défendue par Elliot exclut par principe ces questions.

16 R. Elliot, op.cit., p. 82. 
Ce qui est perdu dans la réplication de la nature qu'autorise la restauration, la plus scrupuleuse soit-elle, c'est bien selon Elliot, la dimension de la genèse, de l'histoire propre d'un site naturel. La naturalité d'un espace est perçue comme l'expression d'une forme de continuité historique, même si elle apparaît circonscrite dans les limites d'un dispositif de protection (comme dans un parc naturel) ou si elle est prise dans une politique de la nature: la continuité des chaînes causales garantit la naturalité. C'est ce qui explique que la réparation des dommages faits à la nature ne soit pas du même ordre que la prévention des dommages: seule la seconde garantit l'authenticité d'une politique aussi bien que l'authenticité de ses résultats. Protéger l'existant est justifié au nom de l'irréversibilité du processus de « dénaturation».

Les choses sont compliquées par le fait que la valeur paysagère d'un ensemble naturel n'est jamais ce qui constitue sa valeur selon les critères de l'éthique environnementale. Pourtant, valeur esthétique et valeur environnementale sont à l'état pratique difficiles à dissocier: les hauts lieux environnementaux sont aussi en règle générale de hauts lieux paysagers. Pour les spécialistes de l'éthique, il importe de rendre possible cette dissociation entre le paysage et la nature. La valorisation de l'état de wilderness ne peut jamais être réduite à une appétence pour la qualité paysagère. Ce qui est valorisé, c'est surtout la compréhension des mécanismes écologiques dont la perception paysagère constitue la traduction ${ }^{17}$. L'intellection l'emporte ici sur l'émotion. Le développement des savoirs écologiques apparaît ici comme une instance de production de nouvelles valeurs : les grilles de lecture de la nature que les biologistes mettent à disposition du public constituent de nouveaux canons de la valeur: sont développés des critères inédits de jugement et d'évaluation des espaces perçus qui contribuent à réorienter le regard sur des espaces anciennement valorisés et surtout à conférer de la valeur à des espaces anciennement stigmatisés ou tenus pour négligeables (c'est le cas déjà évoqué des zones humides). A ce titre, l'écologie n'est pas seulement soumise à une interrogation éthique en tant qu'elle propose des définitions et des styles de traitement de la nature inédits: elle est aussi productrice de valeurs, qui ont nom complexité, diversité, intégration, par exemple. Leur pleine reconnaissance suppose une véritable conversion du regard que nous portons sur la nature.

Si l'on s'en tient au principe de l'irréversibilité stricte de la naturalité, on ne peut pas plus accepter l'idée d'une restauration de la nature que celle d'une restauration de la famille qu'on a perdue: on ne peut pas reproduire, même partiellement, les êtres chers. En conséquence, le génie écologique, qui n'est pas en mesure de reproduire du non reproductible, est une activité du même ordre que toute forme d'aménagement de l'espace: il obéit simplement à d'autres règles de procédure et à d'autres critères d'évaluation. Ceci n'implique pas qu'il soit par définition éthiquement condamnable: si les espaces restaurés ne sont pas donnés pour des morceaux de nature, mais que leur caractère d'artefact est explicité, ils peuvent avoir des fonctions positives (pédagogiques, récréatives et même écologiques). Ces réaménagements de morceaux de nature perdue pourraient être pensés par analogie avec l'encadrement ou l'agrandissement de portraits d'êtres qu'on a perdus, et qui contribuent à entretenir leur souvenir, et donc leur présence

$17 \quad$ R. Elliot, op. cit., p. 87. 
sur un autre mode. Ce qui peut être considéré comme une contrefaçon, c'est dire de ces constructions qu'il s'agit de nature.

Une position radicale dans ce domaine peut consister à ne pas accepter non plus les restaurations qui sont présentées comme telles (et qui ne constituent pas de ce fait des tromperies sur la marchandise ou des contrefaçons, puisqu'on les donne pour ce qu'elles sont), dans la mesure où elles finissent toujours par être confondues avec la nature pleinement naturelle. Je pense qu'on pourrait ici s'aider de la distinction, dans le domaine de l'œuvre d'art, entre le faux (qui constitue une tromperie délibérée) et la copie (qui se donne pour ce qu'elle est et qui peut avoir de nombreuses fonctions positives: diffusion de la connaissance de l'original, support pédagogique, etc.). Une position fondamentaliste conduira dans le domaine de la nature à déclarer illégitimes toutes les copies, dans la mesure où elles sont très peu faciles à distinguer du faux, puisqu'elles partagent les mêmes localisations, in situ. Une position nuancée conduira à reconnaître les mérites de la copie pour toutes les vertus que nous avons évoquées.

\section{3. - NATURALITÉ ET HUMANITÉ}

En fait, le développement d'une problématique de la restauration remet en question la ligne de partage entre naturalité et humanité, et contribue à disqualifier la discussion éthique que je viens d'esquisser. Il ne s'agit jamais pour les défenseurs de l'intervention sur la nature de revendiquer leur action comme l'équivalent de la nature pure: à ma connaissance, on n'utilise jamais pour justifier l'intervention réparatrice l'argument selon lequel des écosystèmes restaurés ou même créés (on pense particulièrement aux lacs) peuvent être, au bout d'un certain temps, visuellement et écologiquement non distinguables d'écosystèmes naturels: on pourrait en effet s'attendre que soit mis en valeur le fait qu'on est désormais en présence d'une dynamique pleinement naturelle, qui s'est trouvée temporairement assistée ou relayée. Nous avons vu qu'à travers les déplacements épistémologiques que suscitait le développement d'une écologie de la restauration la relation entre l'homme et la nature qui est prise généralement comme l'objet de la science des écosystèmes se trouvait sensiblement modifiée: l'homme n'est plus seulement le grand autre de la nature. C'est à partir de son statut de perturbateur (qui lui valait jusqu'à une date récente d'être tendanciellement exclu des scénarios de l'écologie) qu'il est progressivement réintégré dans le jeu à mesure que la nature pure apparaît de plus en plus comme une fiction bien fondée, quelque chose comme un artefact méthodologique.

N'est-il pas significatif de voir Robert Barbault conclure sa communication au colloque du Marais d'Orx qui avait pour thème: «Recréer la nature» en remarquant l'exclusion dont l'homme est l'objet: «N'est-il pas étonnant - et révélateur - que l'on fasse du castor le paradigme de 'l'agenceur écologique' plutôt que l'homme, espèce-clé par excellence... ${ }^{18} \mathrm{~A}$ partir de ce point de vue, la discussion éthique peut changer de base, puisqu'elle tend à se réorganiser autour de l'impé-

18 R. Barbault, Le concept d' espèce clé de voûte en écologie de la restauration : clé ... ou impasse?, «Natures, sciences, sociétés», 1995, n spécial, vol. 3, pp. 19-28. 
ratif du maintien d'un environnement vivable pour l'homme. A ce titre, les objets naturels ne sont pas situables, comme le pense Gunn dans un entre-deux entre la Mitsubishi et la femme adorée (ils seraient alors quelque part entre le statut de produit manufacturable et celui d'être moral). La reconnaissance du caractère non reproductible à volonté de la nature (le fait que les écosystèmes ne puissent être considérés comme des voitures ou des montres), quelles que soient par ailleurs la marge de progrès du génie écologique associé à la génétique et la capacité de fabriquer à terme des «environnements de synthèse», ne conduit pas à étendre aux objets naturels le statut d'êtres moraux, sur le modèle de la position fondamentaliste qui s'incarne dans l'idéologie qu'a développée la deep ecology. Elle définit plutôt les conditions pragmatiques nouvelles d'une maîtrise de l'homme sur la nature qui s'appuie sur un niveau de connaissance accrue des conditions de reproductibilité des ressources naturelles.

On est loin alors de la défense par Aldo Léopold d'une «éthique de la terre», définie comme une extension des frontières de la communauté morale au sol, à l'eau, aux plantes et aux animaux. «Une chose est juste lorsqu'elle tend à préserver l'intégrité, la stabilité et la beauté de la communauté biotique. Elle est injuste quand elle tend à l'inverse», selon l'auteur de l'Almanach d'un comté des sables $^{19}$. L'éthique de la terre constitue sans doute l'exemple le plus radical du décentrement de la morale par l'élargissement de la communauté des êtres en interdépendance, par la mise en avant d'un «sentiment de fraternité avec les autres créatures ». Comme l'ont montré Catherine et Raphaël Larrère, l'éthique de Léopold est une éthique locale, à l'échelle de l'entité écologique que sont la montagne et le marais. L'éthique de Léopold est tributaire de l'écologie de son temps, et elle n'est pas nécessairement adaptée aux questions que pose la problématique de la restauration: le primat de la stabilité, qui fait de la diversité au mieux un moyen d'atteindre à cette stabilité, n'est certainement pas en harmonie avec les développements les plus récents de l'écologie. C. et R. Larrère proposent une intéressante réactualisation de la pensée de Léopold en s'appuyant sur la réinterprétation des travaux de Baird Callicott: «Et si nous inversions les valeurs? Si nous prenions la diversité comme une fin, la stabilité pouvant (ou ne pouvant pas, selon les circonstances), en être le moyen? Serait-ce être infidèle à Leopold, qui ne cesse de décrire et d'aimer la diversité des êtres vivants qui fréquentent ses paysages? Ne montre-t-il pas, à diverses reprises, que la stabilité n'est que relative? Pourquoi ne pas considérer que la traduction contemporaine de la formule discriminante de la land ethic pourrait être: «une chose est juste quand elle tend à préserver (ou augmenter) la diversité biologique. Elle est injuste quand il en va autrement. $»^{20} \mathrm{La}$ position défendue ici transforme largement le point de vue de l'éthique de la terre: l'idée centrale d'un bon usage de la nature développée par les deux auteurs nous rapatrie sur le territoire anciennement balisé d'une éthique de la prudence qui ne suppose qu'une sorte de soumission tactique à la nature, mais qui continue de référer les critères de l'action juste aux exigences de la communauté humaine, fût-elle élargie aux générations futures. Cette extension est impor-

\footnotetext{
19 A. Leopold, Almanach d'un comté des sables, Paris, Aubier, 1995.

20 C. et R. Larrère, Du bon usage de la nature. Pour une philosophie de l'environnement, Paris, Aubier, 1997.
} 
tante pour l'écologie de la restauration aussi bien que pour toutes les politiques de protection puisqu'elle fonde la légitimité de restrictions d'usage pour les contemporains aussi bien que celle des interventions réparatrices. L'élargissement de la communauté morale aux générations futures constitue le principe de justification des politiques de la nature, dont la restauration apparaît ici comme un cas particulier, puisque nous pouvons estimer avoir les moyens de reconstituer partiellement des éléments naturels perdus ou en voie de disparition, parce que nous sommes encore en mesure d'effectuer ces reconstitutions, alors que nous ne sommes pas assurés que nos descendants auront les moyens de le faire. L'obligation à l'égard des générations futures légitime l'intervention.

A la naturalisation de l'éthique défendue par les tenants de la deep ecology s'oppose une historicisation des phénomènes naturels qui a de tout autres implications éthiques. La reconnaissance, plus ou moins partagée de l'historicité de bien des systèmes dits naturels, qui ressemblent fort à ce que sont les habitudes sociales naturalisées dans le domaine des mœurs, dont on a fini par oublier complètement l'origine au point qu'elles apparaissent comme éternelles, a renforcé les effets du repositionnement des écologues autour de problématiques de la gestion des ressources. Les formes d'argumentation éthique sont ici indissociables du statut hybride des restaurateurs de la nature, qui développent une culture professionnelle empruntant à la biologie fondamentale aussi bien qu'à la zootechnie et à l'économie agricole et à des savoir-faire $《$ locaux ${ }^{21}$ : les recompositions éthiques ont toutes les chances de trouver dans ces contextes d'hybridation de théories, de savoir-faire et de tactiques de faire-valoir leur meilleur terrain. Les travaux d'André Micoud et de Valentin Pelosse donnent une idée de la recomposition éthique qui se joue autour de la redéfinition de la partition entre domestique et sauvage. La problématique de la mise en culture du sauvage recoupe bien des questions qui se posent à propos de toutes les interventions réparatrices: c'est bien d'une «redéfinition du statut de l'humanité en tant qu'espèce» qui est en jeu dans l'émergence de nouvelles techniques de production du sauvage, du naturel ou du spontané.

Il semble que l'éthique environnementale place l'écologie de la restauration dans une position contradictoire: si, comme c'est le cas dans les prises de position les plus radicales, toute intervention réparatrice est considérée comme une activité de faussaire, quelle que soit par ailleurs l'ingéniosité des artifices mis en œuvre, l'accent doit être mis exclusivement sur les politiques de protection. Si le principe de diversité est l'axe autour duquel se recomposent les normes éthiques, comme le suggèrent pertinemment $\mathrm{C}$. et $\mathrm{R}$. Larrère, alors la logique de la restauration s'inscrit comme un des éléments principaux de la transmission de l'intégrité d'un patrimoine aux générations futures (ce patrimoine pouvant faire l'objet de placements différenciés, comme la protection et la restauration). Il ne s'agit plus de faire porter l'interrogation éthique sur la légitimité de la restauration en tant que telle, mais plutôt sur ses domaines d'application et sur ses modalités.

On pourrait faire la liste des questions qui sont susceptibles de se poser comme suit:

- le «démiurge» doit-il s'efforcer de s'effacer complètement de son œuvre ou doit-il laisser des traces de son intervention de façon qu'elle puisse être recon-

221 B. Lizet, op. cit., p. 6. 
nue dans sa dimension artefactuelle? Cette question semble primordiale car l'objectif d'une restauration est toujours ambivalent: il s'agit à la fois de restaurer les droits (et la dynamique) de la nature et de choisir entre divers scénarios possibles qui impliquent une part d'arbitraire. Peut-on effacer cet arbitraire relatif?

- peut-on tracer une ligne de démarcation nette entre les exigences scientifiques et les impératifs récréatifs, sachant que ceux-ci constituent un aspect irréductible de toute entreprise viable? Si l'on considère qu'une partie de la productivité éthique de l'écologie de la restauration consiste à convertir le regard à l'appréciation de valeurs proprement écologiques (à travers une appropriation intellectuelle plutôt qu'exclusivement sensible ou esthétique), comment concilier valeur écologique et valeur «paysagère» ou ludique?

- l'écosystème de référence, ou l'écosystème cible (peu importe ici la nuance), doivent-ils être déterminés par les scientifiques eux-mêmes, à l'aide de critères exclusifs à leur discipline considérée dans son état actuel ou doivent-ils faire l'objet d'une négociation préalable avec d'autres acteurs du territoire, qu'ils soient gestionnaires ou simples usagers? Comment organiser dans cette perspective la confrontation des savoirs (scientifiques, techniques, bureaucratiques, savants) susceptibles de s'affronter pour la qualification du territoire?

- comment définir les limites de l'artificialisation et comment sélectionner des techniques d'intervention en tenant compte à la fois de leur efficacité pratique, de leur impact dans la durée, et de leur distance par rapport à des processus «naturels »?

- comment éviter que la possibilité accrue de réparer les dommages de l'anthropisation ne conduise à une gestion imprudente des ressources à l'échelle globale? Comment éviter l'effet pervers que constitue la possibilité croissante d'avoir recours à un petit démiurge pour réparer la nature? Comment éviter l'illusion de la réversibilité?

Cet ensemble de questions peut constituer un bon programme de recherche: il est clair que la biologie de la conservation offre un intérêt particulier pour l'analyse du processus de redéfinition de l'état de naturalité.

L'écologie savante fait de plus en plus droit à l'historicité de ses propres objets, comme d'ailleurs d'autres secteurs de la biologie. Le terrain de la restauration est sans doute celui sur lequel la biologie et les sciences sociales sont amenées à faire des rencontres qui ne soient pas seulement protocolaires. La reconnaissance de la multiplicité des échelles d'observation aussi bien que l'affirmation du caractère «mortel» des configurations décrites installent ces savoirs dans une zone d'incertitude, les rapprochent du statut épistémologique que nous conférons généralement aux sciences sociales à la lecture des Essais sur la théorie de la science de Max Weber ou du Raisonnement sociologique de Jean-Claude Passeron. Il est de plus en plus couramment admis que les scientifiques peuvent proposer des réponses - ou à tout le moins des scénarios - multiples à une question unique: il n'est pas rare qu'ils affirment être dans l'impossibilité de fournir une description univoque d'un processus. S'il existe très probablement un 
décalage entre l'image publique de l'activité scientifique (et partant un système d'attentes sociales) et ce que ces sciences fabriquent actuellement, il n'en reste pas moins que les débats concernant les enjeux environnementaux et leur gestion par la puissance publique contribuent à transformer progressivement la définition sociale de ce qu'on est en droit d'attendre de la production scientifique. Les échanges en cours sur le principe de précaution sont un bon exemple de ce déplacement. Les aspects immédiatement éthiques ou politiques de la discussion ne doivent pas dissimuler ses enjeux proprement épistémologiques: la mise en avant de l'incertitude n'est pas tant la reconnaissance d'une forme d'impuissance du savoir rationnel qu'une redéfinition de ses critères de productivité.

Ecole des Hautes Etudes en Sciences Sociales

SHADYC/CNRS

Marseille 